\title{
Model for radiation damage-induced grain subdivision and its influence in U3Si2 fuel swelling
}

Abderrafi M. Ougouag, Bojan Petrovic, Matias G. Marquez

November 2017

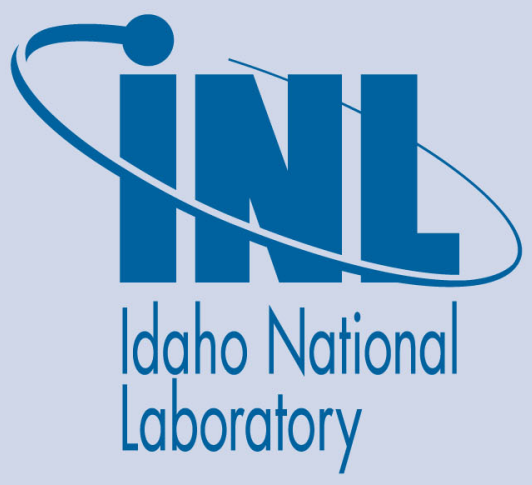

The INL is a U.S. Department of Energy National Laboratory operated by Battelle Energy Alliance 


\title{
Model for radiation damage-induced grain subdivision and its influence in U3Si2 fuel swelling
}

\author{
Abderrafi M. Ougouag, Bojan Petrovic, Matias G. Marquez
}

November 2017

Idaho National Laboratory Idaho Falls, Idaho 83415

http://www.inl.gov

Prepared for the

U.S. Department of Energy

Under DOE Idaho Operations Office

Contract DE-AC07-05ID14517 


\title{
Model for radiation damage-induced grain subdivision and its influence in $\mathrm{U}_{3} \mathrm{Si}_{2}$ fuel swelling
}

\author{
Matias G. Marquez ${ }^{1,2}$, Abderrafi M. Ougouag, ${ }^{3, *}$ and Bojan Petrovic ${ }^{1}$ \\ ${ }^{1}$ Nuclear and Radiological Engineering, Georgia Institute of Technology, Atlanta, GA 30332 \\ ${ }^{2}$ Present address: INVAP S.E., Av. Cmte. Luis Piedrabuena 4950 (R8403CPV) S.C. de Bariloche, Río Negro - Argentina \\ ${ }^{3}$ Idaho National Laboratory, MS 3860, P.O. Box 1625, Idaho Falls, Idaho 83415, USA \\ ${ }^{*}$ Corresponding author, abderrafi.ougouag@inl.gov, +1(208)526-7659
}

\begin{abstract}
The swelling mechanisms of $\mathrm{U}_{3} \mathrm{Si}_{2}$ from neutron irradiation under power reactor conditions are not unequivocally known. The limited experimental evidence that is available suggests that the main driver for swelling in this material would be fission gas accumulation at crystalline grain boundaries. The stages that lead to the accumulation of fission gases at these locations are multiple and complex. However the main mechanism is that, gradually, the gaseous fission products migrate by diffusion. Upon reaching a grain boundary, which acts as a trap, the gaseous fission products accumulate thus leading to formation of bubbles and hence to induced swelling. Therefore, a quantitative model of swelling requires a correct accounting for the presence of grain boundaries and for phenomena and changes that increase their numbers, thus creating sites for bubble formation and growth while concurrently decreasing grain sizes, thus decreasing the effective distance for gas species migration to gas accumulation sites.

A model for the subdivision, or recrystallization, of crystal grains is presented. It is assumed that new grain boundary formation results from the conversion of stored energy from accumulated dislocations into energy for local matter rearrangements that create the new grain boundaries hence subdividing the initial grain. The model is applied to grain subdivision in $\mathrm{U}_{3} \mathrm{Si}_{2}$, albeit using highly uncertain parameters.. Then the implications of the model are assessed using a swelling modeling code to evaluate the total swelling of a fuel pellet during its lifetime in the $I^{2} S-L W R$ reactor, accounting for the influence of grain subdivision.

The present model relaxes assumptions from previous models that limit their applicability in the case of the power levels and temperature conditions of the $\mathrm{I}^{2} \mathrm{~S}$-LWR concept. In particular, the new model assumes fully time-dependent conditions and does not postulate equilibrium or steady-state conditions for any population of radiation induced damage structures or the products of their evolution, such as vacancies, interstitials, and dislocations. Unlike others, the present model explicitly accounts for a population of diinterstitials. The model predicts that when a critical fission density is reached, the crystal grains subdivide and new, smaller grains are formed.

It is shown that for some plausible, though uncertain, sets of physical parameters, a critical fission density can be reached at which recrystallization can occur in $\mathrm{U}_{3} \mathrm{Si}_{2}$, which can then result in the onset of breakaway swelling. It is found that the critical fission density depends strongly on the fission rate density and that it decreases with increases in the fission rate density. The critical fission density is found to display a minimum at intermediate temperatures, which suggest possible fuel performance advantages in operating the reactor in ways that imply higher fuel temperatures.
\end{abstract}

Keywords: fuel recrystallization; fuel swelling model; breakaway swelling; $\mathrm{U}_{3} \mathrm{Si}_{2}$; crystal grain subdivision; dislocations; critical fluence; critical fission density; accident tolerant fuels; rim effect; high-burnup structure

\section{Introduction}

In the context of study of accident-tolerant fuels [1], the feasibility of using $\mathrm{U}_{3} \mathrm{Si}_{2}$ pellets in novel reactors, such as the $\mathrm{I}^{2} \mathrm{~S}$-LWR reactor, must be examined. $\mathrm{U}_{3} \mathrm{Si}_{2}$ is proposed as an advanced fuel for future power reactors to take advantage of its high Uranium density, an attractive feature that allows higher reactivity loading and hence longer fuel cycles [2]. Specifically, in the case of the $I^{2} S-L W R$, the increased Uranium load would allow the use of Stainless Steel as cladding material despite its higher capture cross-section compared to that of Zircaloy, without affecting the duration of fuel in-core residence between reloads [3]. A significant remaining question about the use of $\mathrm{U}_{3} \mathrm{Si}_{2}$ in a power reactor such as the $\mathrm{I}^{2} \mathrm{~S}-\mathrm{LWR}$ pertains to the uncertainty concerning the performance of this fuel under neutron irradiation at the high temperatures typical of power reactors. Such a performance is not known and is, so far, unsatisfactorily modeled, in contrast to the performance of this same fuel under low-temperature research reactor conditions.

Swelling behavior of this fuel is the main concern about its performance under neutron irradiation conditions typical of an operating power nuclear reactor. The extent of swelling, or conversely, the resistance to swelling, is an important factor in determining the fuel lifetime within the core. Significant swelling may lead to fuel and cladding failure, and thus would limit the acceptable residence time within the core. In contrast, a swelling-resistant fuel would allow higher burnup levels and long residence times between refueling outages. This paper 
addresses the modeling of swelling in the $\mathrm{U}_{3} \mathrm{Si}_{2}$ fuel under $\mathrm{I}^{2} \mathrm{~S}$ LWR conditions. A major assumption of existing models for swelling of reactor fuel under neutron irradiation is that swelling is primarily driven by volatile fission products accumulation at grain boundaries or similar structures and by the formation and growth of gas bubbles $[4,5]$. Such models depend heavily on the presence and density of grain boundaries and on the average distance gas and other volatile fission products must migrate to reach such structures. Therefore, the initial focus of this paper is the modeling and prediction of formation of grain boundaries through grain subdivision and the concomitant decrease in the distance fission gas species must migrate to said boundaries. Then, the consequence of higher densities of grain boundaries is evaluated through the use of a publicly available fuel swelling model.

Several theories have been developed in the past three decades to explain or predict the onset of grain subdivision in irradiated materials, especially in $\mathrm{UO}_{2}[6,7,8,9,10]$. Experimentally, it was observed that a new structure forms in the rim of the fuel pellets where original grains could not be identified anymore or where grains of reduced size could be seen. This effect was attributed to high burnup levels, high fission rates and low temperatures. The latter is indeed plausible, as lower temperatures are expected at the periphery of the pellet as it is the closest region of the pellet to the coolant. Furthermore, higher than average fission density and fission rates are also expected at the periphery as the neutron flux is depressed in the inner region of the pellet because of the well-known spatial self-shielding phenomenon (as amplified by resonance self-shielding and transmutation effects) [11].

The motivation for the extensive study of high burnup structures has historically been associated with their effect on fission gas release and on thermal conductivity. However they were not considered an important factor in swelling models, as the rim of restructured material is usually superficial (though some works show a second band of recrystallized grains in $\mathrm{UO}_{2}$ at a deeper location within the pellet [12]) and its effect on global swelling is negligible. Nevertheless, it is possible that for materials different from $\mathrm{UO}_{2}$, such as $\mathrm{U}_{3} \mathrm{Si}_{2}$, the rim may extend to a deeper radial position when high burnup levels are experienced.

Originally, the rim effect was presumed to only be associated with $\mathrm{UO}_{2}$ and $\mathrm{MOX}$ fuels, however, later on, evidence of grain subdivision was found in other types of fuel, supporting the theory that $\mathrm{U}_{3} \mathrm{Si}_{2}$ may go through the same transformation. Moreover, ion irradiated $\mathrm{U}_{3} \mathrm{Si}_{2}$ was found to recrystallize (i.e., experience grain subdivision) [13].

The ability of properly predicting grains subdivision is important because grain boundaries are preferential sites for bubble nucleation and coalescence. Indeed, as grains subdivide, more grain boundaries are created and more bubbles can nucleate there. These bubbles are responsible for gaseous fission fragment-induced swelling.

The approach chosen to model grain subdivision is the formation of dislocation networks composed of dislocation loops that grow and arrange within that structure. These networks consist of walls of dense tangled dislocations surrounding a volume of almost strain-free material (i.e., with no dislocations). These subgrains, with low misorientation between each other, are nuclei for recrystallized grains, with higher misorientation and a larger size, but still much smaller than the original grain size.

The diameter of the dislocation network cells has been extensively studied for several materials, and especially after cold-working in metals. High dislocation densities appear in plastically deformed materials. When the material is heated, the dislocations recover, rearranging and recombining, to minimize their energy. If the recovered material is further annealed, it recrystallizes, as new grain boundaries are formed, dividing the original grains $[14,15,16]$.

The phenomenon previously described is not completely understood, even in mechanically deformed materials [17]. For this reason, difficulties are encountered in attempting to model it in irradiated materials. In this latter endeavor, two key values that the model must predict are the fission density at which grains subdivide and the recrystallized grain size, as both parameters affect swelling calculations.

Obtaining the values just mentioned requires the creation of a phenomenological model that describes the microstructural evolution under neutron irradiation (or equivalently for fuel, upon the accumulation of fission events or upon increase in burnup). Several models were proposed based on reaction rate kinetics where balance equations are used for the creation and annihilation of the different defects. In older models some approximations are generally imposed that assume a steady state is established, as they consider that some species are fast to reach equilibrium during neutron irradiation. However, this assumption is relaxed in the present work.

Another assumption that is generally accepted is that of suppression of the spatial gradients from the differential equations, equivalent to assuming that defects are homogeneously distributed [18]. Although this assumption is not relaxed, some corrections are applied to partly take spatial effects into consideration.

Finally, once the grain subdivision model is applied, its implications are used in the FASTGRASS code [5] to demonstrate the effect of grain subdivision on the rate of the swelling induced by gaseous fission products.

Prior state-of-the-art $\mathrm{U}_{3} \mathrm{Si}_{2}$ swelling models are based (explicitly or implicitly) on very low temperature and high 
fission rate conditions $[7,19,20]$, which seem inapplicable to power reactor conditions since under such conditions the fuel temperature is higher than the critical amorphization temperature, which is defined as the temperature above which amorphization is not possible (i.e., above which damage production and annealing in collision cascades are in equilibrium). Ion irradiated $\mathrm{U}_{3} \mathrm{Si}_{2}$ above amorphization temperature provides evidence of the existence of such a threshold temperature [13].

\section{Experimental background}

The rim effect was first reported by Bleiberg and his coworkers in 1962 [21]. Since then, multiple post irradiation analyses of high burnup reactor pellets have been conducted to characterize the high burnup structure formed in the periphery of the fuel lumps or pellets. Numerous theories have been proposed to explain why the rim effect occurs, however an unequivocally proven theory does not exist. It could be concluded that grain subdivision is caused by several effects acting on the fuel microstructure, making the development of a proper exhaustive model a very demanding task of unrealistic proportions.

Various authors report values for recrystallizing fission densities, recrystallizing temperature, recrystallizing diameters and width of the rim that vary over a broad range. The difficulties associated with measuring or predicting accurate values for fission density (or burnup) and for temperature, as well as the variation of these parameters over time, make it hard to assign a precise set of values for these variables to the observations. Hence, the use of these data for validation of any model should be carried out with great care and caution. The model developed here intends to reproduce the trends observed in other works, without trying to fit the results to data points.

Walker et al. [22], Cunningham et al. [23], Thomas et al. [11], Nogita and Une [24, 25, 26], Matzke [27], and Ray et al. [28] carried out extensive PIE work with pellets showing grain subdivision in the rim. They concluded that subdivision would appear at burnup levels above $60 \mathrm{MWd} / \mathrm{kgU}$ and temperatures below an upper limit between $750^{\circ} \mathrm{C}$ and $850^{\circ} \mathrm{C}$ [26]. It was also determined in those works that the recrystallizing front advances toward the center of the pellet with increasing fission density. Yet, these values tend to vary from work to work (e.g., Lassmann et al. estimate the upper limit for the temperature to be about $\left.1100^{\circ} \mathrm{C}[29]\right)$.

It is consistently assessed that the increase of the burnup toward the pellet edge has a significant effect on the localization of the subdivided band. Therefore the local burnup seems to be the appropriate quantity to relate with the phenomenon, rather than the average burnup. It is estimated that the burnup in the rim of the pellet is approximately twice the average [25].
Another parameter that was measured is the diameter of the recrystallized grains. A wide range of sizes was found, from tens of nanometers to micrometers. However, the average and most probable diameters were between $100 \mathrm{~nm}$ and $500 \mathrm{~nm}$ $[11,26,27,29]$.

With respect to swelling and grain subdivision in $\mathrm{U}_{3} \mathrm{Si}_{2}$, data are scarce; and most of the literature refers to temperatures below the amorphization critical temperature. Furthermore, only one report was found in which swelling of irradiated bulk (non-dispersion) crystalline $\mathrm{U}_{3} \mathrm{Si}_{2}$ is measured [30]

\section{A model for radiation-induced grain subdivision}

\subsection{Description}

The model created in the present work is based on the one proposed by Rest and co-workers for grain subdivision in uranium-based fuels [7, 9, 31]. That model was subsequently used to predict the high swelling observed in $\mathrm{U}_{3} \mathrm{Si}_{2}$ dispersion fuels. However, later on, it was superseded by a model that explained swelling as attributed to bubble growth in an amorphous material. The initial model, though, was used to explain grain subdivision in the rim of $\mathrm{UO}_{2}$ pellets, where grains are supposed to eventually subdivide under neutron irradiation. The present work applies the initial model to $\mathrm{U}_{3} \mathrm{Si}_{2}$, but now with the added consideration that neutron irradiation occurs at a temperature above the critical amorphization temperature (i.e., the fuel will not become amorphous at any dose). The new model also relaxes the postulate of the establishment of a steady state (see below) for some important physical quantities and allows grain subdivision to occur during the transient phase of the microstructure evolution. Another change from Rest et al.'s original model is the introduction of a new criterion for determination of onset of recrystallization: the new approach is based on physical considerations and a mathematical formulation that are similar to the description of steady-state creep. Additional differences between Rest's model and the present one are enumerated and briefly discussed below.

The present model consists of two main moduli: a set of differential equations that describe the temporal evolution of the fuel microstructure and a second modulus that evaluates the fission density at which the material recrystallizes and the resulting size of the newly formed (smaller) grains. The set of differential equations (described below) defines the evolution of the concentration of vacancies, interstitials, di-interstitials, interstitial loops (and average diameter) and dislocation networks.

Although Rest's model is proposed in terms of a set of differential equations (as emulated in the present work), it applies approximations to use steady state values for most of 
the defects concentrations. This latter assumption seems to work well for Rest's applications, but the present work proposes a different way of obtaining the fission density, and consequently the grain size, at which the material recrystallizes. An important modification introduced in the present work is to require an explicit accounting for the temporal evolution of the microstructure, making the new model applicable under true transient conditions. This modification is introduced because the recrystallization point is thought to belong to a state of the microstructural evolution at which equilibrium has not yet been reached. This a-priori insight is justified a-posteriori as computations using the new model show recrystallization occurs long before a state of equilibrium or a steady state is attained. An incidental, though non-negligible, benefit of this modification is to allow an explicit treatment of true transient conditions, such as variable irradiation histories or time-dependent fission densities.

Another modification made to Rest's model is the inclusion into the set of equations of one for the evolution of the diinterstitials concentration. This type of defect can be considered a "buffer" as its concentration expresses the balance between its creation and consumption events, however with a special property: if a di-interstitial disappears because it grows into a larger defect or coalesces into an interstitial loop population, it cannot return to the di-interstitial population. This assumption can be confidently accepted, as the stability of interstitial complexes grows with their size. Taking into account the di-interstitials separately helps to define an average diameter for the interstitial loops in a better, more precise, way. This is because in the absence of an explicit separate accounting for di-interstitials, inclusion of their impact on the overall population of interstitial loops would require a reduction of the overall average interstitial loop size without a proper physical reason. Indeed, the alternative of accounting for the effect of the small size of di-interstitials without explicitly tracking their number would require reducing the average size of all interstitial loops without a proper physical reason.

To further improve the accuracy of the model, the population of interstitial loops could be divided into several size segments to obtain a size distribution; however this consideration would make the calculation very expensive and render the model too unwieldy for the scope of this work.

Another theory added to the original model is that of "defect clustering." Various authors have studied this phenomenon to improve the modeling of the microstructural evolution when collision cascades resulting from neutron irradiation (and fission events) create heterogeneous damage, and vacancies and interstitials group (or cluster) in a segregated fashion. The distribution of point defects thus generated has three main effects when compared to the traditional damage model in which homogeneously distributed Frenkel pairs are created: (i) high recombination rate in the cascade neighborhood, (ii) vacancy and interstitial clusters formation and (iii) temperature dependence of the previous two items due to vacancy emission from vacancy clusters at higher temperatures. The main consequence of the defect clustering phenomenon is a reduction in the concentration of free migrating point defects released to the medium by each cascade event. A secondary effect is the formation of interstitial clusters that can nucleate to form interstitial loops. As an approximation, these clusters are assumed to contribute to the di-interstitial production; however, in reality, clusters of different sizes can form.

From the above considerations one recognizes an important contribution to the extended defect evolution from the production of small interstitial loops in the cascade, which then grow into the dislocation network. Without this consideration, the interstitial loops would only be created from the evolution of freely migrating interstitials.

As the set of differential equations is solved to yield the temporal evolution of the microstructure, an energy balance is performed at each time step to assess the feasible recrystallizing diameter, which is defined as the diameter at which the energy necessary to create new grain boundaries is offset (or just exceeded) by the stored energy in the dislocation networks. However, this definition is considered incomplete as such a balance would always be true for some particular recrystallization diameter. Therefore another constraint had to be added with a relationship of the number or amount of subgrains that rearrange to constitute each recrystallized grain. This relationship was assumed to be similar to the five-power law for creep (see further discussion later, in the text describing Equation (20), within the present paper)

\subsection{Model governing equations}

Firstly, two equations describing the evolution of the point defects (vacancies and interstitials) are postulated:

$$
\begin{gathered}
\frac{d C_{v}}{d t}=Q_{v}-K_{i v} C_{v} C_{i}-K_{v N}\left(\rho_{N}\right) D_{v} C_{v} \\
-K_{v L}\left(\pi C_{I} d_{I}\right) D_{v} C_{v}-k_{g b}^{2} D_{v} C_{v} \\
-\frac{4 \pi a_{0}^{2} D_{v} C_{v} C_{2 i}}{\Omega} \\
\frac{d C_{i}}{d t}=Q_{i}-K_{i v} C_{v} C_{i}-K_{i N}\left(\rho_{N}\right) D_{i} C_{i}-K_{i L}\left(\pi C_{I} d_{I}\right) D_{i} C_{i} \\
-k_{g b}^{2} D_{i} C_{i}-16 \pi r_{i v} D_{i} C_{i}^{2} \\
+\frac{4 \pi a_{0}^{2} D_{v} C_{v} C_{2 i}}{\Omega}
\end{gathered}
$$

where $C_{v}$ is the concentration of vacancies, $C_{i}$ is the concentration of interstitials, $\rho_{N}$ is the dislocation network density, $C_{2 i}$ is the concentration of di-interstitials, $Q_{v}$ is the generation rate of free migrating vacancies, $Q_{i}$ is the generation rate of free migrating interstitials, $K_{i v}$ is the recombination rate 
for interstitials and vacancies, $\Omega$ is the atomic volume (an average value computed as in [10]), $a_{0}$ is the lattice parameter, $b_{v}$ is Burgers vector, $D_{v}$ is the diffusion coefficient for vacancies and $D_{i}$ that for interstitials.

The generation rate of free migrating vacancies, $Q_{v}$, and the generation rate of free migrating interstitials, $Q_{i}$, are related to the fission rate as [7]

$$
Q_{v, i}=\frac{\dot{f}}{B}
$$

where $\dot{f}$ is the fission rate and $B$ is the reciprocal of the particular defect species production efficiency from fission events, in effect a factor that relates the two quantities $Q_{v, i}$ and $\dot{f}$. Obviously, the larger the value of $\mathrm{B}$, the lower the generation rate of the particular species. Eventually $Q_{v}$ and $Q_{i}$ could be different, as when the effect of production bias is considered [32]; however for simplicity of the model, and due to lack of information on this subject, bias is neglected in the present work.

The recombination rate can be calculated per

$$
\mathrm{K}_{\mathrm{iv}}=\frac{4 \vartheta \pi D_{i} r_{i v}}{\Omega}
$$

where $r_{i v}$ is the distance of interaction between vacancies and interstitials. Theoretically, $\vartheta$ should be equal to 1 , but due to some approximations that are used, it can take a value around 10 according to Was [4].

The sink strength of the grain boundaries $\left(\mathrm{k}_{\mathrm{gb}}^{2}\right)$ is calculated as follows:

$$
\mathrm{k}_{\mathrm{gb}}^{2}=\frac{24}{d_{g}^{2}}
$$

where $d_{g}$ is the grain diameter. $K_{j N}\left(\rho_{N}\right)$ and $K_{j L}\left(\pi C_{I} d_{I}\right)$ represent the sink strength of the dislocation networks and the dislocation loops for $\mathrm{j}$ (with $j=i, v$ ), respectively. These two functions were evaluated as $Z_{j N} \rho_{N}$ and $z_{j L} \pi C_{I} d_{I}$, respectively. The coefficients $z$ are calculated the same way as by Dubinko et al. [33].

The first four terms in the right sides of Equations (1) and (2) represent the same physical processes: the generation rate of the point defects, the recombination rate of point defects, the annihilation of point defects by aggregation into the dislocation network and the annihilation of point defects upon their reaching grain boundaries, respectively. The last term in eq. (1) represents the loss of vacancies through reaction with diinterstitials, while the fifth and sixth terms of eq. (2) represent the loss of interstitials through the formation of di-interstitials and the release of an interstitial when a di-interstitial reacts with a vacancy.

A second set of model-governing equations represents the evolution of the extended defects:

$$
\begin{gathered}
\frac{d C_{2 i}}{d t}=Q_{2 i}+8 \pi \frac{r_{i v} D_{i} C_{i}^{2}}{\Omega^{2}}-4 \pi \frac{r_{i v} D_{i} C_{v} C_{2 i}}{\Omega}-\frac{\pi v_{2 i} C_{2 i}}{b_{v}} \\
\frac{d \rho_{N}}{d t}=f_{1} \frac{\left|v_{D}\right|}{d_{N}} \pi d_{I} C_{I}-f_{2} \frac{\left|v_{N}\right|}{d_{N}} \rho_{N} \\
\frac{d C_{I}}{d t}=\frac{\pi v_{I} C_{2 i}}{2 a_{0}}-\frac{4 v_{I} C_{I} d_{I}}{d_{N}^{2}}-8 v_{I} d_{I}^{2} C_{I}^{2}-f_{1} \frac{\left|v_{D}\right|}{d_{N}} C_{I} \\
\frac{d\left(d_{I}\right)}{d t}=v_{I}-\left(d_{I}-2 a_{0}\right) \frac{C_{2 l \rightarrow I}^{\cdot}}{C_{I}}-\left(d_{N}-d_{I}\right) \frac{C_{I \rightarrow N}}{C_{I}} \\
-d_{I} \frac{C_{2 I \rightarrow I_{2}}}{C_{I}}
\end{gathered}
$$

where $C_{21 \rightarrow \mathrm{I}}^{\cdot}, C_{\mathrm{I} \rightarrow N}^{\cdot}$ and $C_{2 I \rightarrow I_{2}}^{\cdot}$ are the rates of transformation of di-interstitials into interstitial loops, interstitial loops into dislocation networks and the rate of coalescence of interstitial loops, respectively. These are given by

$$
\begin{gathered}
C_{2 l \rightarrow I}^{\cdot}=\frac{\pi v_{2 i} c_{2 i}}{2 a_{0}} \\
C_{I \rightarrow N}^{\cdot}=\frac{4 v_{I} C_{I} d_{I}}{P_{s l} d_{N}^{2}} \\
C_{2 I \rightarrow I_{2}}^{\cdot}=8 v_{I} d_{I}^{2} C_{I}^{2}
\end{gathered}
$$

There is no need to add a differential equation to describe the diameter of the dislocation network, as it is directly related to the dislocation network density by

$$
d_{N}=C_{A} C_{\rho} \sqrt{\frac{\pi}{f_{v} \rho_{N}}}
$$

where $C_{A}$ and $C_{\rho}$ are two geometric parameters and $f_{v}$ depends on Poisson's Ratio (v) according to

$$
\mathrm{f}_{v}=\frac{1-\frac{v}{2}}{1-v}
$$

The values of $C_{A}$ and $C_{\rho}$ are not completely known, but Molecular Dynamics simulations obtain a value around 1.4 for the product of the two of them [34].

$v_{I}$ is the dislocation climb speed and it is given by 


$$
v_{I}=\frac{2}{b_{v}}\left(z_{i} D_{i} C_{i}-z_{v} D_{v} C_{v}\right)
$$

where $z_{i}$ and $z_{v}$ are the dislocation sink strength for interstitials and vacancies, respectively. These parameters are calculated using an approximate expression given by Dubinko et al. [33]; $v_{2 i}$ is the climb speed of di-interstitials. It is approximated as

$$
v_{2 i} \approx \frac{1}{2 b_{v}} D_{i} C_{i}
$$

Solving these equations, it is possible to obtain $C_{v}, C_{i}, C_{2 i}$, $\rho_{N}, C_{I}, \mathrm{~d}_{\mathrm{N}}$ and $\mathrm{d}_{\mathrm{I}}$ as functions of neutron irradiation time and use them as an input for the subsequent part of the model where the recrystallization diameter is calculated. For this latter calculation, a balance is set up where the available energy (i.e., the stored energy in the dislocation networks) is compared to the energy required to create a new grain boundary. This procedure yields the diameter of the hypothetical recrystallized grain [7] as

$$
d_{c r x}=\frac{3 \gamma_{G B}}{\Delta E_{S}}
$$

where $\gamma_{G B}$ is the grain boundary surface energy and $\Delta E_{S}$ is given by [15]

$$
\Delta E_{S}=\frac{\rho_{N} G b_{v}^{2} f_{v}}{4 \pi} \ln \left(\frac{C_{A} C_{\rho}}{d_{N} \rho_{N} b_{v}}\right)+\frac{G}{2}\left(\frac{b_{v} f_{v} d_{N} \rho_{N}}{2 \pi C_{A} C_{\rho}}\right)^{2} .
$$

In this evaluation, not only is temperature dependence used for the shear modulus $(G)$, but also dependence on burnup is included to take into account a decrease in the elastic modulus observed as a consequence of burnup, which in fuel correlates with neutron irradiation [35]. Then, the shear modulus can be expressed as

$$
\begin{aligned}
\mathrm{G}=\mathrm{G}^{0}\left(1-1.09154 \times 10^{-4} T\right)[0.9 \\
\left.-\frac{2}{\pi} \frac{0.1}{0.905} \operatorname{atan}\left(\frac{\dot{f} t-5 \times 10^{-26}}{2.5 \times 10^{-26}} \frac{20}{3}\right)\right]
\end{aligned}
$$

where $\mathrm{G}^{0}$ is the shear modulus of unirradiated (e.g., $\mathrm{UO}_{2}$ ) at a reference temperature, $T$ is the temperature in Kelvin and $\dot{f}$ is the fission rate density in fissions $/ \mathrm{sec}-\mathrm{m}^{3}$, the instantaneous time derivative of the fission density. An approximate value for the reference shear modulus, $G^{0}$, is found in the paper by Marlowe [36]. A nearly identical value is inferred from the value of Young's modulus at $\mathrm{T}=0$ using the relevant equation from Reference [31] and assuming isotropic behavior. The value given in Table 1 is approximately the mean of the two values just mentioned. The temperature dependence shown in the above equation is the same as that of Young's modulus given in Reference [31].

At last, a final equation is added that estimates the number of subgrains that recrystallize into a single new grain. It is assumed that recrystallization depends on the temperature and on the stress generated by the dislocations. This phenomenon has some similarity with that of creep; therefore an analogous equation to that of creep is used. Thus, the five-power law equation for steady state creep is adapted in such a way that the number of subgrains within a newly formed grain would be proportional to a creep-like five-power expression. Furthermore, the diameter of the recrystallized grain would have to be proportional to the cubic root of the number of subgrains that coalesce therein. Then, it can be shown that the following expression is suitable for calculating the number of subgrains that form a grain:

$$
\mathrm{n}_{\text {subg }}=\left(\frac{C \sigma_{N}^{5} D_{S I A}}{T}\right)
$$

In the above formula, $C$ is a constant that can be parametrically adjusted, $\sigma_{\mathrm{N}}$ is the average stress around the dislocation network and $D_{S I A}$ is the diffusion coefficient of self-interstitial atoms. From equation (18), it can be demonstrated that

$$
\sigma_{\mathrm{N}} \approx K b_{v} \sqrt{\rho_{N}}
$$

Also, the radiation enhanced diffusion coefficient of a selfinterstitial atom can be evaluated as

$$
\mathrm{D}_{\mathrm{SIA}}=C_{v} D_{v}+C_{i} D_{i}
$$

Then, using Equations (21) and (22) in Equation (20), one finds that

$$
\mathrm{n}_{\text {subg }}=\frac{\tilde{C}\left(b_{v} \sqrt{\rho_{N}}\right)^{5}\left(C_{v} D_{v}+C_{i} D_{i}\right)}{T}
$$

This leads to a "thermally" activated recrystallization critical diameter given by

$$
d_{\text {crit }}= \begin{cases}d_{N} & n_{\text {subgr }}<1 \\ d_{N} \sqrt[3]{n_{\text {subgr }}} & n_{\text {subgr }} \geq 1\end{cases}
$$

Using equations (17) and (24), it is possible to find the critical dislocation density and the recrystallization diameter. The critical fission density is obtained from the neutron irradiation time step at which these values are attained. 


\subsection{Di-interstitial production rate}

As explained above, the heterogeneous distribution of vacancies and interstitials that exists after a collision cascade has cooled down gives rise to clusters of interstitials, which in turn lead to the formation of interstitial loops. This effect can be incorporated into the first term of the right side of equation (6).

Similarly, vacancy clusters also form. And at high temperature they emit vacancies that can react with the interstitial clusters thus reducing the effective production and production rate of di-interstitials. Further work is needed to model this effect; however that is out of the scope of this paper. Yet, based on the results seen in other works $[37,38]$, the diinterstitial production rate can be modeled as

$$
Q_{2 i}=Q_{2 \mathrm{i}}^{0} P_{m}
$$

where $P_{m}$ is the probability for an interstitial cluster to nucleate into a loop and $\mathrm{Q}_{2 \mathrm{i}}^{0}$ is the amount of interstitial clusters produced per fission event.

In addition to the equations shown above, it is implicit that other equations apply that quantify the diffusion coefficients for interstitials and vacancies as a function of the appropriate jump frequencies and migration activation energies. Such equations can be found in any standard textbook that covers solid state diffusion.

\section{Results}

Based on the theory presented in this paper, a code has been written that solves the coupled differential equations and that finds the critical recrystallization fission density and the recrystallization diameter. The two main variables that change within the pellet are the temperature and the fission rate. Therefore, the mathematical model is solved for different combinations of temperature and fission rate. However, some physical parameters are unknown, which implies an increase in the number of simulations that are needed to carry out a comprehensive parametric study.

The fission rate is varied from typical PWR average values to higher fission rates comparable to the ones found in the rim of fuel pellets. For temperature, the values range between hypothetical surface and centerline temperatures.

\subsection{Model Validation for $\mathrm{UO}_{2}$}

After adjusting the parameters that were not known, it became possible to predict the rim effect with some degree of accuracy.
As previously stated, a three-year neutron irradiation, corresponding to the shortest intended in-core fuel life design, was simulated using different temperatures and fission rates. (The three year limit is chosen arbitrarily though it could be considered a cutoff below which the fuel concept might be non-competitive.) Then, the evolution of the microstructure was analyzed to find if recrystallization occurs using the recrystallization criterion detailed above. The parameter $\tilde{\mathrm{C}}$, from equation (23), is varied until the two curves depicting the recrystallization diameter (the curve obtained from the energy balance and the one obtained considering the amount of subgrains that recrystallize into one new grain) intersect, thus defining the critical fission density at which recrystallization takes place. This parameter, $\tilde{\mathrm{C}}$, is not changed when modeling similar cases in which only different temperatures and fission rates are used.

The physical properties used in the model for $\mathrm{UO}_{2}$ are detailed in Table 1 and Table 2. In Table 1, values of parameters with low level of uncertainty are displayed. In Table 2, estimates for the more uncertain parameters are listed.

Table 1. Known parameters for $\mathrm{UO}_{2}$.

\begin{tabular}{|c|c|c|c|c|c|}
\hline \multicolumn{3}{|c|}{ Measured } & \multicolumn{3}{|c|}{ Calculated } \\
\hline $\begin{array}{l}\text { Physical } \\
\text { property }\end{array}$ & Value & Ref. & $\begin{array}{l}\text { Physical } \\
\text { property }\end{array}$ & Value & Ref. \\
\hline $\begin{array}{c}\mathbf{G}^{\mathbf{0}} \\
(\mathbf{G P a})\end{array}$ & 80 & {$[34]$} & $\begin{array}{c}r_{i v} \\
(\mathbf{n m})\end{array}$ & 8.2 & {$[8]$} \\
\hline $\boldsymbol{v}$ & 0.31 & [34] & $\begin{array}{c}\mathbf{b}_{\mathbf{v}} \\
(\mathrm{nm})\end{array}$ & 0.382 & [31] \\
\hline $\begin{array}{c}a_{0} \\
(\mathbf{n m})\end{array}$ & 0.541 & {$[6]$} & $\begin{array}{c}\Delta V_{v} \\
\left(\mathbf{n m}^{3}\right)\end{array}$ & -0.002 & [34] \\
\hline $\begin{array}{c}\gamma_{G B} \\
\left(\mathbf{J} / \mathbf{m}^{2}\right)\end{array}$ & 1 & [31] & $\begin{array}{c}\Delta V_{i} \\
\left(\mathbf{n m}^{3}\right)\end{array}$ & 0.006 & [34] \\
\hline $\begin{array}{c}\Omega \\
\left(\mathbf{n m}^{2}\right)\end{array}$ & 0.0427 & [9] & $C_{A} C_{\rho}$ & 2.185 & [34] \\
\hline
\end{tabular}

All the parameters in these tables, and in Tables 3 and 4, are defined elsewhere in this paper, except for $v_{0}^{v}, \epsilon_{v}^{m}, v_{0}^{i}$ and $\epsilon_{i}^{m}$ that stand for the jump frequencies and migration activation energies for vacancies and interstitials, and for $\Delta V_{v, i}$ that stand for the relaxation volume of vacancies $(v)$ and interstitials $(i)$, respectively. These latter parameters enter in the Dubinko formulation mentioned above. 
Table 2. Estimated parameters for $\mathrm{UO}_{2}$.

\begin{tabular}{|c|c|c|}
\hline \multicolumn{3}{|c|}{ Estimated } \\
\hline $\begin{array}{l}\text { Physical } \\
\text { property }\end{array}$ & Value & Ref. \\
\hline $\begin{array}{c}\epsilon_{\mathrm{v}}^{m} \\
(\mathrm{eV})\end{array}$ & 2.4 & [39] \\
\hline $\begin{array}{c}\epsilon_{\mathrm{i}}^{m} \\
(\mathrm{eV})\end{array}$ & 0.6 & [39] \\
\hline $\begin{array}{c}v_{v} \\
\left(\mathbf{s}^{-1}\right)\end{array}$ & $1 \times 10^{13}$ & [40] \\
\hline $\begin{array}{c}v_{i} \\
\left(\mathbf{s}^{-1}\right)\end{array}$ & $1 \times 10^{14}$ & [40] \\
\hline B $\left(1 / \mathrm{m}^{3}\right)$ & $10^{23}$ & a \\
\hline
\end{tabular}

${ }^{a}$ value of $\mathbf{B}$ for which $6 \%$ of point defect produced survive as free migrating defects.

Using the SRIM code [41] the number of atomic displacements created by a fission event was computed. Assuming 5 to $6 \%$ of these survive the early phase damage cascade formation, the damage production efficiency per fission event for interstitials and vacancies, $B^{-1}$, was computed.

Using these parameters, a solution was obtained that approximates the behavior of the fuel rim. Figure 1 shows curves depicting the resulting critical fission density at different temperatures for different fission rates. The curves are only plotted for the range within which subdivision occurs; absent (such as for the fission rate of $1.510^{19} \mathrm{fission} / \mathrm{s} . \mathrm{m}^{3}$ ) or cropped curves mean that the points that are not plotted correspond to states for which subdivision does not occur. Hence, it can be observed that for low fission rates (e.g., 1.5 $10^{19}$ fission $/ \mathrm{s} . \mathrm{m}^{3}$ ), crystalline grains remain undivided over the whole range of temperatures or only subdivide within a small range $\left(1.7510^{19}\right.$ fission $/ \mathrm{s} . \mathrm{m}^{3}$ case). However, as the fission rate increases, the temperature range for which sub-division occurs broadens and the critical fission density at a given fixed temperature decreases. Another feature of interest in Figure 1 is the shape of the various curves, as all appear to show a decrease as the temperature increases from low values, before showing a reversal and increases as the temperature moves into high values. The curves seem to bottom at around $950 \mathrm{~K}$. This model result is expected, as two different underlying limiting mechanisms dominate the behaviors at low and high temperature, respectively, while at intermediate temperatures neither is as effective at limiting or inhibiting the advent of subdivision or recrystallization. Specifically, at low temperatures the mobility of dislocations is hindered, while at high temperatures damage recovery is enhanced. Both of these mechanisms, in their respective ways, cause an increase in the fission density needed to reach a state at which a sufficient density of dislocations has built up to cause recrystallization.

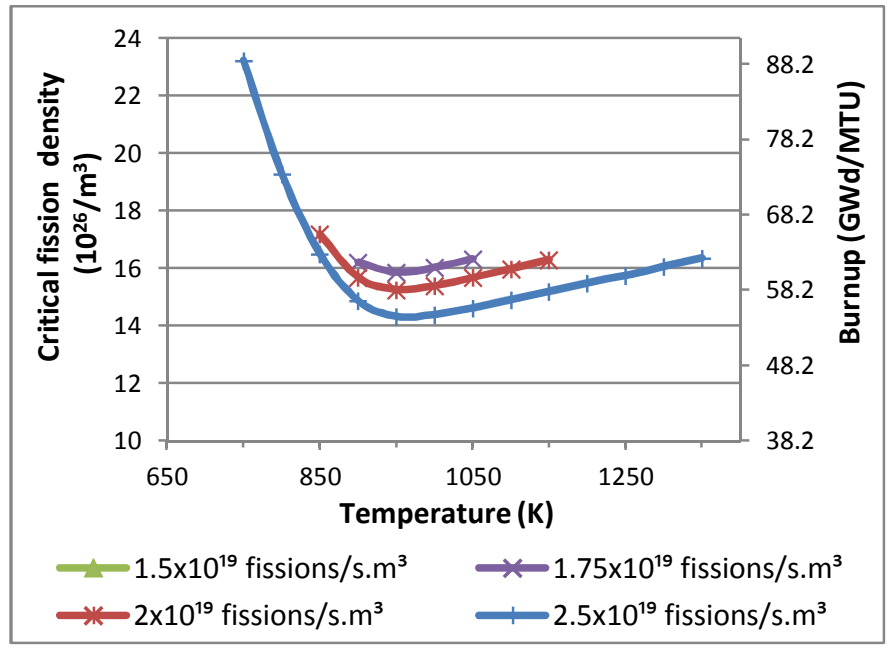

Figure 1. Critical fission density vs. temperature for different fission rates for $\mathrm{UO}_{2}$.

At the intermediate temperatures the mobility is enhanced in comparison to the situation at low temperatures, while annealing of damage is significantly lower than what it would be at higher temperatures. Hence, the curves exhibit a minimum indicating that, at the intermediate temperature of around $950 \mathrm{~K}$, an overall lower fission density is sufficient for causing recrystallization.

Also, it is possible to obtain the recrystallized grain diameter as the diameter at which conditions for recrystallization are realized and hence the grain subdivides. The values of the recrystallized diameter are plotted in Figure 2 for four fission rates as the temperature is varied, in a way similar to the critical fission density of Figure 1. Again, the results are plotted only when recrystallization occurs (hence the curve is inexistent for the $1.510^{19}$ fission $/ \mathrm{s} . \mathrm{m}^{3}$ fission rate case). If for a certain temperature and a given fission rate the grains do not recrystallizes, no point is available to be shown in the plot.

Although the model performs well in predicting the rim effect, the concentration of loops that is computed is higher than expected. This could be considered a flaw of the model; however state-of-the-art models for the behavior of the microstructure exhibit the same higher than expected loops concentration [42]. This means that the models overestimate the production rate of loops and underestimate the rate at which loops join the network. Although this feature appears to be inconsequential for the prediction of recrystallization, further work is being performed to devise a model without this imperfection. 


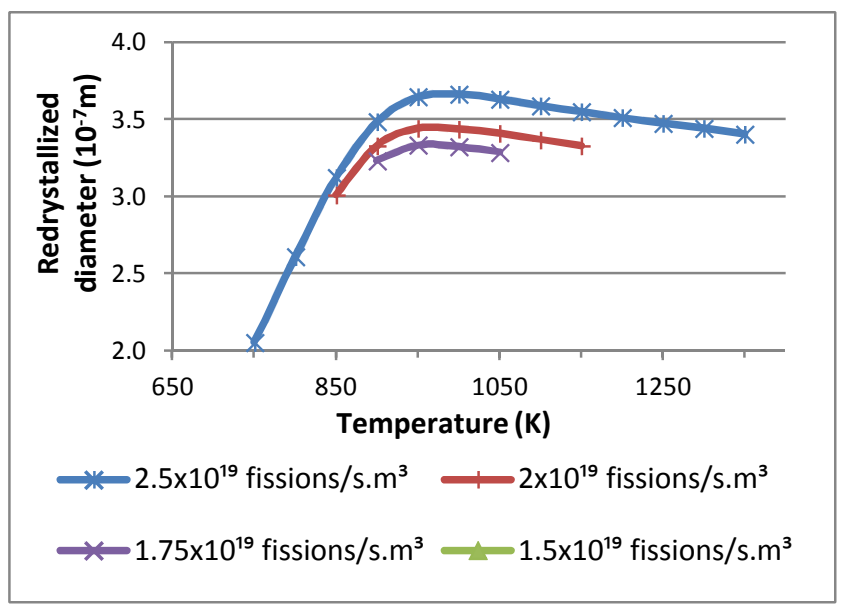

\section{Figure 2. Recrystallization diameter vs. temperature for different fission rates for $\mathrm{UO}_{2}$.}

It is interesting to note that the effect of variations in the migration activation energies and jump frequencies is negligible (estimated parameters listed in Table 2). This can be expected since the evolution of the extended defects is mainly driven and characterized by the products $D_{v} C_{v}$ and $D_{i} C_{i}$, which are predominantly determined by radiation enhanced diffusion and tend to stabilize, at constant temperature, at the same value, whatever the diffusion coefficient.

\subsection{Grain subdivision in $\mathrm{U}_{3} \mathrm{Si}_{2}$}

Having obtained reasonable results from the model, when compared to the observed rim effect in high burnup $\mathrm{UO}_{2}$, the model is applied in an attempt to predict the performance of $\mathrm{U}_{3} \mathrm{Si}_{2}$. This is accomplished by changing the model parameters from those of $\mathrm{UO}_{2}$ to parameters assumed applicable and relevant to $\mathrm{U}_{3} \mathrm{Si}_{2}$. However, the number of unknown parameters for $\mathrm{U}_{3} \mathrm{Si}_{2}$ is much greater than that for $\mathrm{UO}_{2}$. For this reason, it is difficult to obtain a conclusive solution that reliably represents actual physical behavior in the absence of experimental data to corroborate the model and to provide the means for adjusting unknown parameters. Yet, some physical insight can be gained by varying the unknown parameters within physically reasonable ranges. Therefore, the effect of varying different parameters, especially those that suffer greater uncertainty, is studied. Using the model, a solution is obtained that is similar to the one for the $\mathrm{UO}_{2}$ case. This solution is derived using the same constant for the amount of subgrains that recrystallize into a new grain, a parameter that can only be determined experimentally given the current state of the art of microstructure evolution models. The known and the estimated parameters used in the model are detailed in Table 3 and Table 4, respectively.
Table 3. Known parameters for $\mathrm{U}_{3} \mathrm{Si}_{2}$.

\begin{tabular}{|c|c|c|c|c|c|}
\hline \multicolumn{3}{|c|}{ Measured } & \multicolumn{3}{|c|}{ Calculated } \\
\hline $\begin{array}{l}\text { Physical } \\
\text { property }\end{array}$ & Value & Ref. & $\begin{array}{l}\text { Physical } \\
\text { property }\end{array}$ & Value & Ref \\
\hline $\begin{array}{c}\mathbf{G}^{0} \\
(\mathbf{G P a})\end{array}$ & 33 & [30] & $\begin{array}{c}\mathbf{a}_{0} \\
(\mathbf{n m})\end{array}$ & 0.34 & [7] \\
\hline \multirow[t]{4}{*}{$v$} & 0.17 & [30] & $\begin{array}{c}\Omega \\
\left(\mathrm{nm}^{3}\right)\end{array}$ & 0.14 & [9] \\
\hline & & & $\begin{array}{c}\mathbf{b}_{\mathbf{v}} \\
(\mathbf{n m})\end{array}$ & 0.382 & a \\
\hline & & & $\begin{array}{c}\Delta V_{v} \\
\left(\mathbf{n m}^{3}\right)\end{array}$ & -0.0308 & $\mathrm{~b}$ \\
\hline & & & $\underset{\left(\mathrm{nm}^{3}\right)}{\Delta V_{i}}$ & 0.252 & $\mathrm{~b}$ \\
\hline
\end{tabular}

Table 4. Estimated parameters for $\mathrm{U}_{3} \mathrm{Si}_{2}$.

\begin{tabular}{|c|c|c|}
\hline \multicolumn{3}{|c|}{ Estimated } \\
\hline $\begin{array}{l}\text { Physical } \\
\text { property }\end{array}$ & Value & Ref \\
\hline $\begin{array}{c}\epsilon_{\mathrm{v}}^{m} \\
(\mathrm{eV})\end{array}$ & 0.9 & [7] \\
\hline $\begin{array}{c}\epsilon_{\mathrm{i}}^{m} \\
(\mathrm{eV})\end{array}$ & 0.3 & $\mathrm{c}$ \\
\hline $\begin{array}{c}v_{v} \\
\left(\mathbf{s}^{-1}\right)\end{array}$ & $1 \times 10^{13}$ & [40] \\
\hline $\begin{array}{c}v_{i} \\
\left(\mathrm{~s}^{-1}\right)\end{array}$ & $1 \times 10^{14}$ & [40] \\
\hline $\begin{array}{c}r_{i v} \\
(\mathbf{n m})\end{array}$ & 8.2 & d \\
\hline $\begin{array}{l}\gamma_{G B} \\
\left(\mathbf{J} / \mathbf{m}^{2}\right)\end{array}$ & 0.5 & - \\
\hline $\begin{array}{c}\text { B } \\
\left(1 / \mathbf{m}^{3}\right)\end{array}$ & $10^{23}$ & $\mathrm{e}$ \\
\hline$C_{A} C_{\rho}$ & 2.185 & d \\
\hline \multicolumn{3}{|c|}{$\begin{array}{l}{ }^{\mathrm{c}} \text { Rough estimation via multiplying } \\
\text { the } \mathrm{UO}_{2} \text { value by a number close to } \\
\text { the ratio of the } \mathrm{U}_{3} \mathrm{~S}_{\mathrm{i} 2} \text { melting } \\
\text { temperature to that of } \mathrm{UO}_{2} \\
{ }^{\mathrm{d}} \text { Same value as } \mathrm{UO}_{2} \text { used. } \\
{ }^{\mathrm{e}} \text { value of } \mathbf{B} \text { for which } 6 \% \text { of point } \\
\text { defect produced survive as free } \\
\text { migrating defects. }\end{array}$} \\
\hline
\end{tabular}

As can be seen in Figure 3, the fuel recrystallizes only at very high fission rates (on the order of the rates at the rim). Also, there are an upper and a lower temperature limits between which recrystallization occurs and beyond which, on either side, it does not. The model solution is plotted for temperatures ranging from $550 \mathrm{~K}$ to $1400 \mathrm{~K}$, yet points (and 
lines between them) are effectively displayed only within a portion of this temperature range.

This indicates that subdivision only occurs within the range of temperatures for which a line is visible. A similar behavior was also observed in the previous figure.

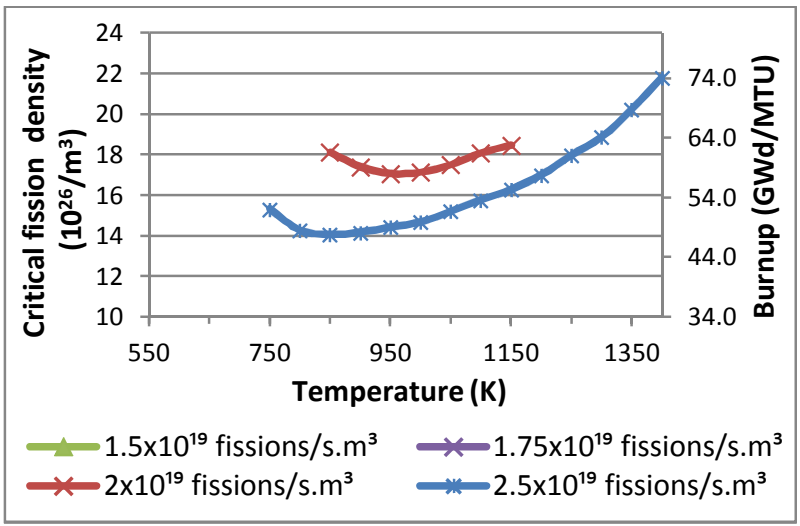

Figure 3. Critical fission density vs. temperature for different fission rates for $\mathrm{U}_{3} \mathrm{Si}_{2}$.

The results just discussed assume that the dependence of the shear modulus on burnup is similar to that of $\mathrm{UO}_{2}$. If this correction is not assumed, the solution that is obtained is the one shown in Figure 4.

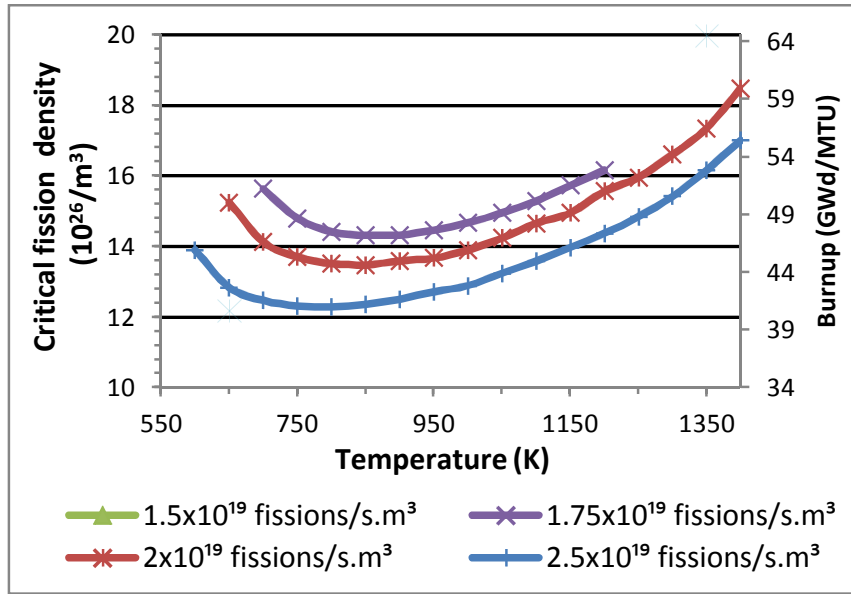

Figure 4. Critical fission density vs. temperature for different fission rates for $U_{3} S_{3}$ without effect of burnup on shear modulus.

It is apparent from the figure that this correction makes an important difference on the critical fission density. At low fission rates, i.e., rates comparable to the average value in the bulk of the fuel pellet, the fuel is not predicted to recrystallize by the present model. This behavior would be advantageous if upheld upon a future experimental verification.

From both Figure 3 and Figure 4 it can be seen that the temperature dependence of the critical fission density implies that at the single-pellet level recrystallization would be more prevalent at the periphery than in the inside within the bulk of the pellet. This would be the case as the outer rim of the pellet is expected to be colder than the inside because of proximity to the coolant and the physics of heat transfer and therefore an overall lower critical fission densities would be needed to cause recrystallization. This differential effect would be amplified by the spatial self-shielding effect, as the outer pellet region would experience higher neutron fluxes and hence higher burnup levels than the inner region.

In both Figure 3 and Figure 4 the behavior previously discussed in conjunction with Figure 1 can be observed again. Specifically, the lower mobility of dislocations at low temperatures and the enhanced damage recovery at high temperatures result in larger values of the critical fission density for onset of recrystallization, while at intermediate temperatures recrystallization requires comparatively lower fission densities. The consequent seemingly parabolic shape of the curves is believed to be a fortuitous result of the model. Indeed, the curves are the result of solving over 18 coupled non-linear ordinary differential equations, and a simple mechanism that would suggest a parabolic behavior versus temperature cannot be readily inferred, especially in light of the different mechanisms at play at low and high temperatures, respectively, as discussed above. The seemingly parabolic behavior is merely the reflection of a change in dominant mechanisms as the temperature is varied from the lower range to the higher one.

Another feature of the temperature dependence of the critical fission density exhibited in Figure 3 and Figure 4, besides the existence of a temperature at which a minimum occurs, is the shifting of said temperature of the minimum as the fission rate (i.e., specific power) is varied. Specifically, as the specific power is raised the minimal critical fission density decreases and occurs at lower temperature. However, the most important feature of the figures is the decrease of the minimum critical fission density as the volumetric fission rate is increased.

The decrease in the minimum value of the critical density is easily understood in terms of rate effects common in radiation effects. As the damage rate (or defect formation rate) increases, unless balanced by a commensurate increase in damage recovery rate, damage efficiency (i.e., fraction of surviving damage structures, net of prompt recombination) and damage asymptotic saturation levels would increase and hence the populations of various damage structures would increase merely because the strength of the source that gives rise to them is increased. In the context of the present model, such behavior translates into the rise or occurrence of conditions for recrystallization at an overall lower fission density as the fission rate increases. 
The decrease in the temperature of the minimum can similarly be explained by rate effects: as the fission rate increases, so do the density of initial defects and their formation rate. Hence, there also results and increase in the density of dislocations sufficient to compensate for lower mobility at lower temperatures, which in turn causes the critical fission density to occur at lower temperature than otherwise.

The occurrence of the minimum between 800 to $950 \mathrm{~K}$ or 800 to $850 \mathrm{~K}$, depending on whether the shear modulus dependence on burnup is accounted for or not, respectively, suggests that operating the reactor away from these values would result in enhanced fuel performance. Since operating at lower temperatures would carry thermodynamic efficiency penalties, it appears that operating at higher temperatures would be preferable, provided excessive temperatures that would also otherwise threaten fuel integrity (e.g., pellet center meltdown) are avoided. However, before being relied upon, the temperature values provided here must be confirmed through application of the model with more reliable parameters.

Another parameter that has a noteworthy effect on the result is the constant defining the relation between the network dislocation density and the network cell diameter $\left(C_{A} C_{\rho}\right)$. The effect of increasing this parameter results in higher critical fission densities. If one assumes that for $\mathrm{U}_{3} \mathrm{Si}_{2}$ this parameter would be between the value for $\mathrm{UO}_{2}$ and the value for metals, then one can infer that it could be higher than the value used for $\mathrm{UO}_{2}$. Figure 5 shows the change of the critical fission density versus temperature for two different values of $C_{A} C_{\rho}$ and a volumetric fission rate of $1.75 \times 10^{19} \frac{\text { fissions }}{\text { sec. } \mathrm{m}^{3}}$. The $C_{A} C_{\rho}$ constant is doubled from the lower to the upper curve.

It is still necessary to model properly the damage produced by fission fragments in the material. Variations in the damage efficiency, the cluster production rate and the cluster nucleation probability have a considerable effect on the final result. An increase in the magnitude of the freely migrating point defects production rate impacts the radiation enhanced diffusion, increasing dislocation mobility and promoting recrystallization. An increase in the rate of loop nucleation from clusters would raise the population of dislocations and thus would accelerate recrystallization. Figure 6 shows the effect of increasing the amount of freely migrating defects produced per fission event given by a change in the parameter $B$ from equation (3).

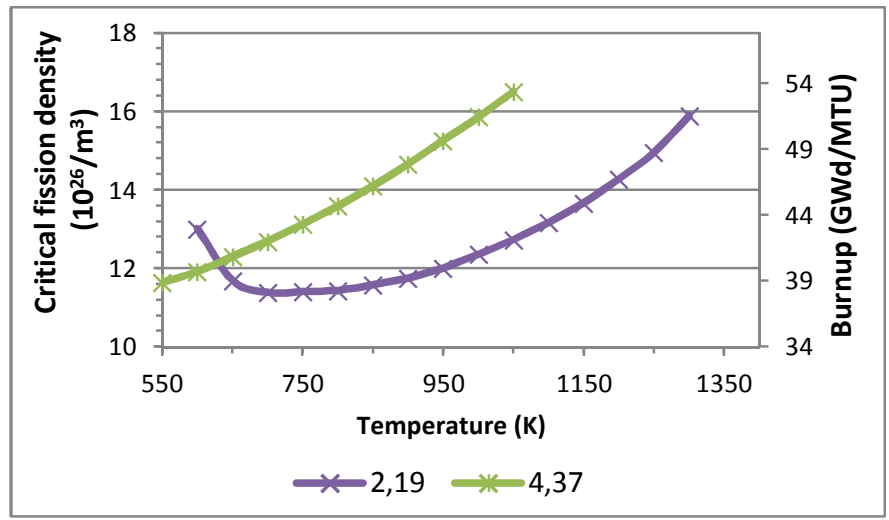

Figure 5. Effect of doubling the parameter $C_{A} C_{\rho}$

Larger rates of production (corresponding to the lower values of B) imply increased (and earlier, i.e., at lower fission density) recrystallization. It is noteworthy that the case of the largest value of $B$, namely $610^{25} \mathrm{~m}^{-3}$, results in no recrystallization and hence in the absence of a corresponding curve in the figure. Thus, it is meaningless to solve the model for higher values of $\mathrm{B}$, as no recrystallization would be observed.

\subsection{Swelling calculations using FASTGRASS}

The FASTGRASS code [5] was used to quantify the effect of grain subdivision, or recrystallization, on the onset and extent of swelling of bulk fuel material. Several cases were run to span a variety of conditions. However, all cases are hypothetical; as the fission density for onset of crystal subdivision is not computed using the theory presented above, but instead is assumed to occur at some arbitrary point during the neutron irradiation process as burnup of the fuel accumulates. Another hypothetical feature is that when recrystallization is assumed to have taken place, it is postulated to have occurred throughout the pellet, and not solely in an outer rim region. Such hypothetical cases are acceptable and sufficient for the purpose of demonstrating the effect of grain subdivision on the onset and evolution of swelling. Finally, an important feature of the hypothetical models is that subdivision is assumed to occur regardless of the prediction of the recrystallization model. Specifically, in the cases with high temperature (e.g., $1220 \mathrm{~K}$ ), it is assumed that the same level of subdivision occurs as in the other cases, although an examination of Figure 4 would allow one to conclude that for such high temperatures subdivision would occur at much higher burnup levels than in the other temperature cases, if at all. This deliberate decoupling of the crystal grain subdivision and the swelling calculations is intended to prevent any misuse of the results presented here, as explained further in Section 5. 


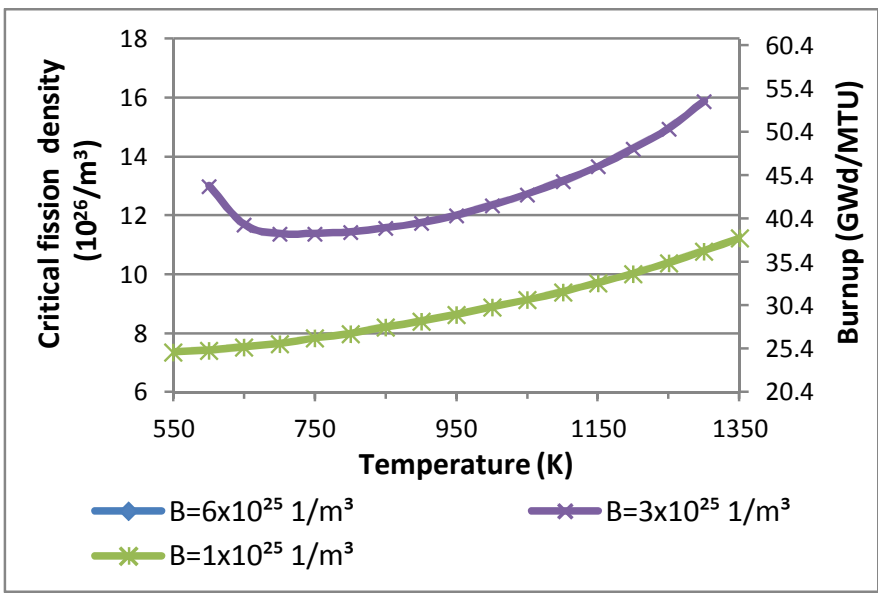

Figure 6. Effect on critical fission density of the amounts of freely migrating defects produced.

A disadvantage of using FASTGRASS to perform these calculations is that the code might not provide a high fidelity estimation of the bubble size given that a large volumetric fraction of the bubbles observed in the rim do not belong to any of the classes considered by the code (lattice, grain face, grain edge). Instead, the rim bubbles may correspond to discrete localized aggregations of part of the released gas, which thus are trapped and do not complete the release process out of the pellet. As most of the porosity in the rim is constituted of this latter type of bubbles, accounting for them requires an unconventional use of the FASGRASS code. Specifically, the effective release probability used as an input in FASTGRASS (represented by the BVCRIT parameter) is reduced until the code predicts a density of grain edge bubbles corresponding to the same pores percentage volume as reported in the literature to be observed in the pellet rim.

Nevertheless, at least in a qualitative manner, it is possible to evaluate the effect of grain size on the migration of gas atoms to the surface of the grains and how the reduction of the grain diameter enhances swelling. It must also be highlighted that the final swelling calculation is independent of the fission density at which the grain recrystallizes, as the two computations are totally decoupled.

The modeling/computational procedure described above is justified, as the bulk of the fission gases produced is still present within the grain lattice prior to grain subdivision. Only when the grain subdivides, and as the path from within the body of the grain to its surface becomes shorter, do the gases find their way out of the lattice to the grain face. The kinetics of the gas species migration out of the grain after subdivision is probably fast, so a short transition between the two states (ingrain/out-of-grain) is observed. This can be inferred from Figure 7, which shows the fractional swelling versus the fission density for two arbitrary cases. The two cases postulate two different (and arbitrary) critical fission densities at which subdivision occurs. Following subdivision, both cases display a sharp relative increase in volume (i.e., swelling), which imply a sharp increase of volatile species migration to the grain faces. This abrupt change in swelling rate, which occurs at the critical fission density, is termed the "breakaway swelling" and is characteristic of the swelling mechanism driven by recrystallization or crystal subdivision. This behavior will be noted in all the figures in this paper that depict swelling performance following recrystalization.

Another important observation from Figure 7is that the final swelling performance is independent of the fission density at which the subdivision occurs. Indeed, the figure shows the fractional swelling for two arbitrary and different recrystallization critical fission density values, yet at higher fission density values the two curves re-join into one.

A final observation is that following subdivision and the initial jump in swelling, the continuing swelling takes place at a significantly higher rate than that observed prior to subdivision (except for the cases of $\mathrm{U}_{3} \mathrm{Si}_{2}$ with medium and high fission gas release, which are discussed later).

From the above observations, the main conclusion is that the FASTGRASS code predicts swelling to follow whenever subdivision is assumed to have occurred regardless of the fission density at which such subdivision is postulated. Of course, in a more complete simulation, the actual critical fission density at which subdivision occurs would have to be determined (per the above model), as it would be the trigger for the swelling rate change.

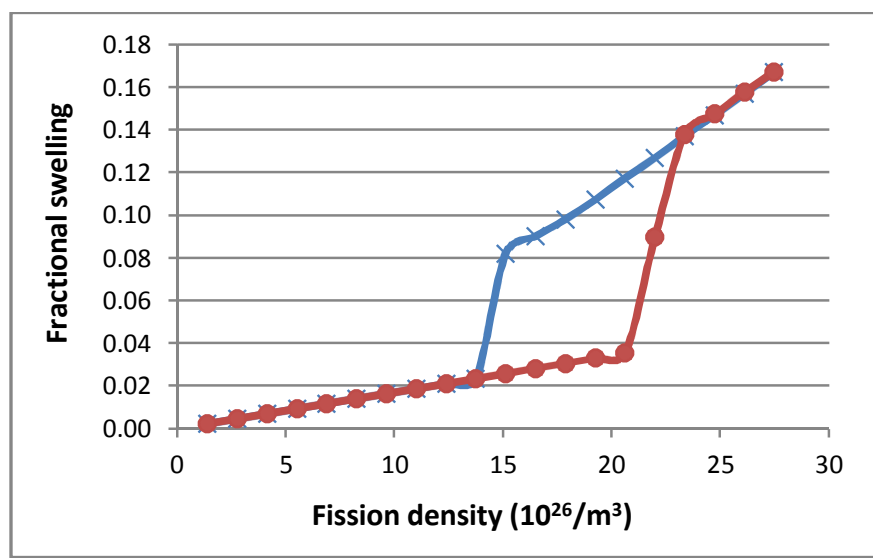

Figure 7. Fractional swelling for two different arbitrary postulated recrystallization fission density values.

Figure 8 shows the effect of temperature on the swelling for recrystallized grains in $\mathrm{UO}_{2}$ with a discharge burnup of 69 $\mathrm{GWd} / \mathrm{MTU}$. In this hypothetical case, the grain subdivision is arbitrarily assumed to occur at the three fourths point of the lifetime of the fuel pellet (51.75 GWd/MTU). Although this value may be too low, as explained above, the amount of final end-of-life swelling seems to be independent of the fission 
density at which the grain subdivides (all other parameters, e.g. temperature, being equal), while, as shown in Figure 7, it does depend on the subdivision having occurred. Also, it must be noted that the subdivision is assumed to occur at the same fission density for all cases that are considered, though it is clear from Figure 4 that subdivision would occur at different fission densities that depend on the fission rate and on the temperature of the material.

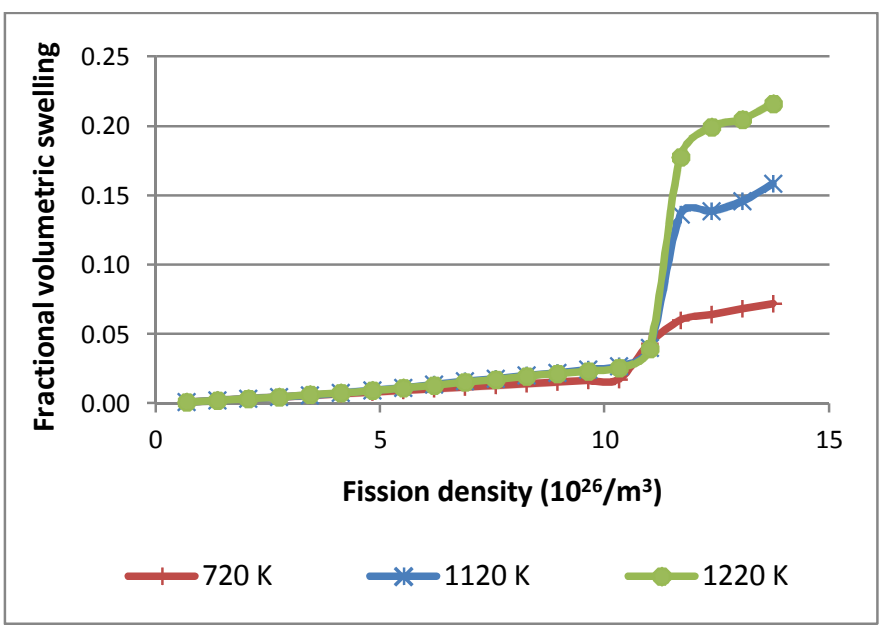

Figure 8. Swelling in UO2 for different temperatures under rim conditions (without fission gas release)

Figure 9 displays a similar hypothetical case as that of Figure 8 , but for $\mathrm{U}_{3} \mathrm{Si}_{2}$. In both cases the sharp rise in swelling corresponds to the effect of subdivision, as it occurs at the burnup level (or fission density) that is postulated in the hypotheses for these cases. Since the swelling is shown as a fractional increase in volume, it is clear that if subdivision occurs throughout the fuel pellet, the overall effective swelling of the pellet would be that shown in the figures. Conversely, if subdivision were to occur only in a rim region, then the overall effective swelling level would be the weighted average of the swelling in the non-subdivided inner region of the pellet and of the large swelling in the rim region. It is thus obvious that the swelling is much higher if all the fuel recrystallizes throughout the pellet.

The swelling values presented in Figure 8 and Figure 9 do not consider fission gas release. This is legitimate, as the experimental evidence from subdivided fuel would imply (for the current model of uniform fission density) that the gases remain in the pellet within large pores and hence contribute to the swelling. If the release of the gases from the pellet is enhanced at high temperature, or even becomes complete, then the computed swelling would be much smaller.

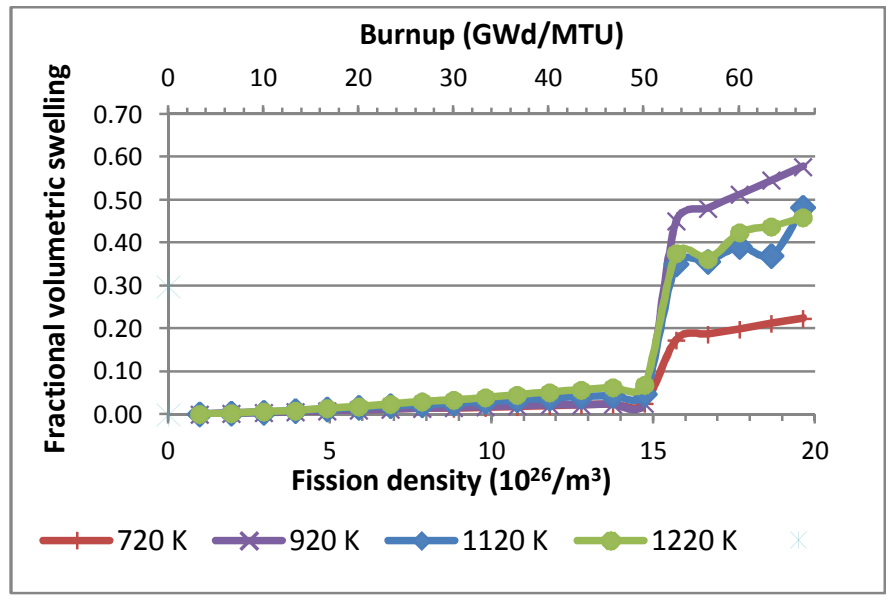

Figure 9. Swelling in $U_{3} \mathrm{Si}_{2}$ for different temperatures under postulated rim conditions. (without fission gas release)

When comparing the swelling of $\mathrm{UO}_{2}$ and $\mathrm{U}_{3} \mathrm{Si}_{2}$ after subdivision, larger swelling values are observed in the latter. This is caused by the lower surface tension in $\mathrm{U}_{3} \mathrm{Si}_{2}$, which leads to higher equilibrium bubble sizes for the same amount of gas at the same temperature and pressure. This may be one of the most important disadvantages of $\mathrm{U}_{3} \mathrm{Si}_{2}$ from the swelling point of view.

If the fuel does not recrystallize, then the swelling calculated using FASTGRASS is much lower, as can be seen in Figure 10.

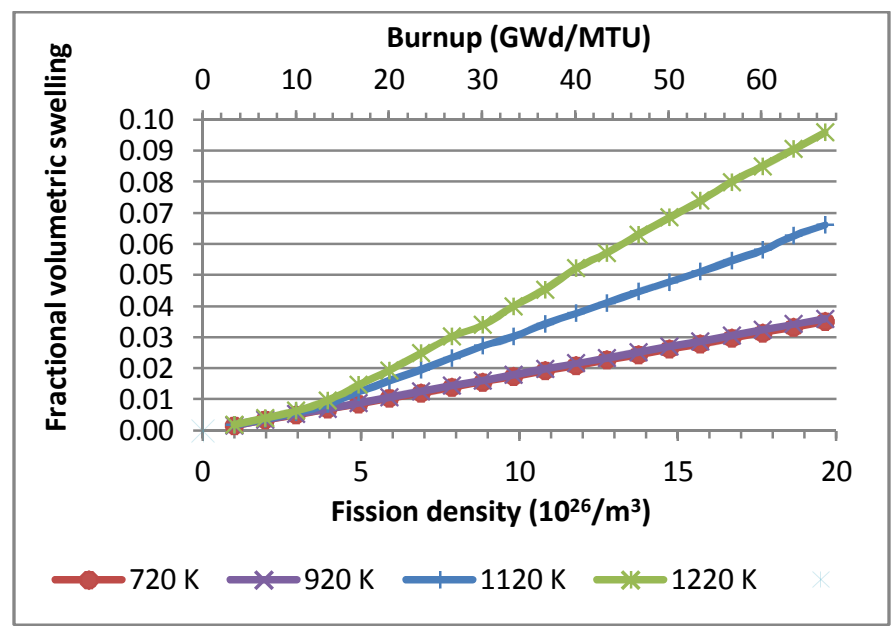

Figure 10. Swelling of $U_{3} \mathrm{Si}_{2}$ without recrystallization.

This behavior indicates that the absence of the additional grain surfaces limits the swelling as the gases remain within the body of the grains. Conversely, the behavior of Figure 8 and Figure 9 shows the swelling performance when fission gases migrate to the more numerous grain boundaries (following subdivision) and form bubbles. A third type of behavior would be one in which the fission gases are released beyond the grain 
boundaries. In this latter behavior it can be recognized that the release of the fission gases has the property of limiting the swelling. If the probability of fission gases to be released is increased, then the swelling would rapidly saturate at low values. Figure 11 shows the swelling for four temperatures setting the FASTGRASS parameter BVCRIT (which determines the critical value of grain edge swelling required for long-range tunnel interlinkage [5]) to 0.05 .

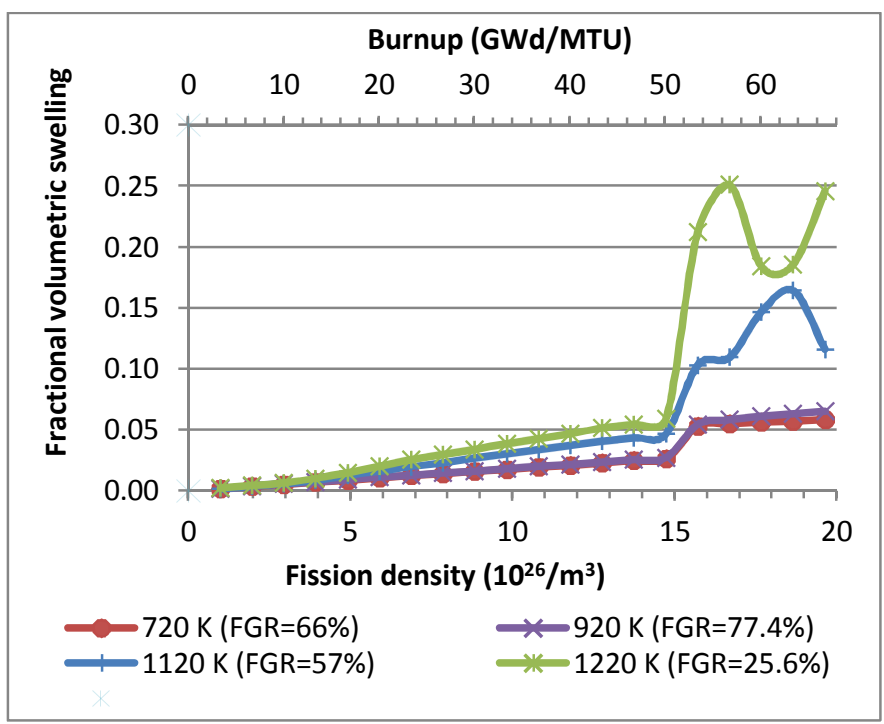

Figure 11. Swelling of recrystallized $U_{3} \mathrm{Si}_{2}$ with postulated high fission gas release.

The swelling values in the last figure are smaller than the ones shown in Figure 9. This shows the importance of correctly predicting the fission gas release, especially at high temperature.

Swelling for a lower, but still not negligible, fission gas release fraction was calculated too. The result can be seen in Figure 12.

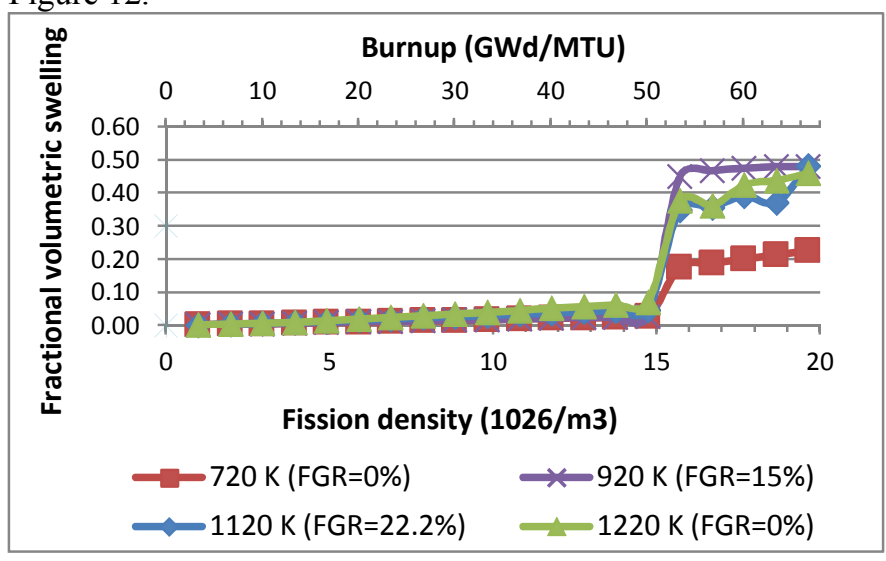

Figure 12. Swelling of recrystallized $U_{3} \mathrm{Si}_{2}$ with postulated medium fission gas release.
A further observation from Figure 8 and Figure 9, as well as Figure 11 and Figure Figure 12, is that although the onset of high-rate swelling coincides in all cases with the subdivision of crystal grains, the subsequent behavior appears to vary from case to case. In the lower temperature cases, the swelling rate changes from low to suddenly large then back to a low rate. In contrast, the higher temperature cases show a markedly different behavior. At first sight, it appears as though the mechanism assumed in FASTGRASS for calculating fission gas migration from the atomic lattice to the grain faces and forward from the grain faces to grain edges might be impacting the behavior of the swelling in an apparently erratic (or nonmonotonous and irregular) way. However, upon closer examination of the highest temperature cases (especially for $\mathrm{U}_{3} \mathrm{Si}_{2}$ ), one may notice that swelling is followed by shrinking then swelling again (and in one case, a repeat shrinking then swelling). Such a behavior does not correlate with any additional microstructural changes that could be predicted by the theory developed in this work and is recognized as the result of phenomena confined within the swelling modeling code. At present, it is uncertain whether the observed behavior is merely the consequence of numerical artifacts or the outcome of interplay between competing processes such as, for example, the possible alternating buildup and partial, thermally enhanced, release of fission gases at microstructural features ranging from the smallest and finest (i.e., within grains) to the coarser (grain edge then onward to crystal edge and plenum). Though a direct verification of this hypothesis is beyond the scope of the present paper, its further examination should be the subject of future work.

Another important observation is that the effect of temperature is not monotonous, which indicates that multiple mechanisms are competing with each other. While for low fission gas releases the swelling peaks at a temperature close to $920 \mathrm{~K}$, if the release probability is increased, that peak decreases in absolute value for the medium release case (note the differing scales of the ordinates axis in the three figures) and finally disappears in the high release case. However, the percentage release at that temperature does not change. This behavior is not fully understood and should be investigated further.

Finally, a word of caution regarding the interpretation and use of the model results is worth repeating. In all the modeled cases of swelling the onset of grain subdivision is postulated to occur at the same hypothetical critical fission density (or critical neutron fluence). Thus, in the examples shown here for illustrative purposes, the swelling behavior would appear to be inconsistent with the recrystallization temperature and fission density dependence results of the first part of this paper (e.g., Figure 4). Of course, there is no contradiction, since in the second part of the paper the onset of recrystallization is assumed to occur at a set fission density and not at a computed critical fission density. Nevertheless, at least for $\mathrm{UO}_{2}$, it was 
demonstrated (in the discussion of Figure 7) that final swelling is independent of the recrystallization fission density.

\section{Discussion and Conclusion}

Modeling of microstructural evolution of fuel under neutron irradiation is one of the major challenges in the construction of fuel performance models. This is even more so for novel fuel forms where only limited experimental data are available. The combined effects of atomic and mesoscale phenomena in highly heterogeneous configurations mandate the introduction of several assumptions and simplifications that deteriorate the fidelity of the models and decrease the accuracy of predictions. Absent conclusive experimental data, analyses at lower scales must be implemented in order to estimate parameters that are used in higher scale model equations. Until either data or lower scale models become available, the lack of consistent and well-known parameters and the uncertainty about details of physical conditions increase the complexity of the validation process.

The importance of accurately predicting the onset of grain subdivision stems from the importance of grain boundaries acting as sites for bubble growth and hence favoring material swelling, as was proven in prior work by others.

Based on these considerations, a rational model has been developed for the formation of subgrains in nuclear fuels due to the grouping of dislocations and their evolution into cell walls and subsequent local material recrystallization, the mechanism underlying fuel swelling under neutron irradiation and giving rise to the characteristic breakaway swelling behavior. The distinguishing features of the present work are that (i) the developed recrystallization model is a transient one in contrast to the previous quasi-static predecessor, (ii) that it relies on much fewer adjustable parameters and (iii) that it adheres to physical models considerations to as wide an extent as possible. The new model predictions were compared to the observations from the subdivided rim of $\mathrm{UO}_{2}$ high burnup fuels, and a satisfactory agreement is observed, essentially validating the model for that application. The model was then used, in an extrapolated way, to estimate the extent of subdivision in $\mathrm{U}_{3} \mathrm{Si}_{2}$. In this latter case, some parameters were arbitrarily chosen, as no constraint or data could be identified that would impose a value or even a specific range of values. For this reason, it is recommended that the model be calibrated and validated using data from irradiated $\mathrm{U}_{3} \mathrm{Si}_{2}$ when such data become available.

The computationally demonstrated significant sensitivity of the model with respect to some of the lesser known parameters in $\mathrm{U}_{3} \mathrm{Si}_{2}$ allows a priority ranking of the parameters that should be estimated using experimental data. In particular, the temperature and fluence dependence of the shear modulus, G, the $\boldsymbol{B}$ and the $C_{A} C_{\rho}$, parameters of $\mathrm{U}_{3} \mathrm{Si}_{2}$ should receive the earliest attention.

It is important to highlight the finding that the recrystallization front will probably advance from the colder part of the fuel pellet to the hotter because the former reaches the critical fission density sooner than the latter (because of spatial self-shielding). This is an advantage as, all other conditions being equal, the consequence of recrystallization on swelling is lower at lower temperatures since the same amount of gaseous species would occupy smaller volumes and exert lower outward pressures on the materials surrounding them. Furthermore, the rate of migration of gaseous species to grain boundaries would be lower at low temperatures, hence lowering the rates of nucleation and growth of bubbles. Hence, the thickness of the rim would have to be unusually large if it were to reach into zones with temperatures at which said rim could considerably affect swelling. For completeness, it is worth mentioning that at much lower temperatures, typical of research reactors, the literature shows that a different mechanism altogether appears to dominate [9].

Other considerations at the level of the pellet could be used to support the development of operational guidelines. Indeed, different performance zones can be defined within a pellet, using temperature and burnup as criteria. Intermediate temperatures (near $850 \mathrm{~K}$ ) and high burnup promote grain subdivision, and consequently, high swelling (rim conditions). The rest of the pellet experiences a higher temperature (and lower burnup). For these latter conditions, grain subdivision is more difficult, though still possible. To prevent fuel restructuring from occurring all across the pellet, the average burnup should be kept below a threshold that the present model could predict once the unknown parameters are properly estimated. A possible practical engineering solution would be to aim for average burnup levels lower than the threshold and to operate at temperatures as high as possible while not exceeding an acceptable temperature limit beyond which the risk for pellet melting would rise unacceptably (a requirement favored by the high thermal conductivity of silicide fuel, which flattens the within-pellet temperature profile). In seeking such an operating temperature threshold, it is noteworthy that it appears that working temperatures above $1200 \mathrm{~K}$ may prevent grain subdivision, as suggested by some of the figures in this paper. However as for other conclusions, uncertainty will prevail until the model is tuned using experimental data.

Another consideration regarding operating at higher temperature is that at higher temperature,it is possible that grain growth might compensate for the grain size reduction caused by recrystallization. If this were indeed to occur, compared to the situation without compensation, a lower density of grain boundaries would result and hence fewer bubble nucleation and growth sites. Concurrently, gaseous species migration from within grains bulk to the grain boundaries would be impeded, as the average diffusion 
distances would be effectively larger. These two behaviors would amount to a reduction of the influence of recrystallization on bubble formation and growth and on the concomitant implication on swelling. The mitigating effect of higher temperatures may be further amplified if hightemperature-enhanced fission gas release were to lead to gases escaping the pellet entirely or to be trapped within larges pores present inside the pellet instead of migrating to grain boundaries. However, while fission gas release would contribute to alleviating swelling, it would also lead to potential fuel performance and design concerns, such as, for example, the need for an increased fuel rod plenum volume. Obviously, both of these temperature-induced phenomena should be further modeled and/or evaluated through experiments.

Caveat Emptor: The model presented in this paper is fully suitable for assessing or predicting the performance of $\mathrm{UO}_{2}$ fuel pellets. In contrast, the same is not true for $\mathrm{U}_{3} \mathrm{Si}_{2}$ and could not be expected to be so until corroborated by experimental evidence when it becomes available and until the model parameters are determined either through experiments or first principles-based modeling. For this reason, in this paper the prediction of crystal subdivision or recrystallization and the demonstration of the influence of recrystallization on swelling were deliberately decoupled from one another. That is to say, the microstructural model of subdivision, once developed, was not used to compute the amount of recrystallization as an input to the swelling model (although that was possible to carry out). The demonstration of the relevance of recrystallization to subsequent swelling was achieved with assumed recrystallization at an arbitrary fission density. This choice was made in order to discourage the use of the numerical results in actual design applications for $\mathrm{U}_{3} \mathrm{Si}_{2}$, as the model parameters for this material are highly uncertain and model results cannot be considered reliable until experimental data

\section{References}

1. Lahoda, E.J. and F.A. Boylan, Development of LWR Fuels with Enhanced Accident Tolerance; Final Technical Report. RT-TR-15-34, 432 p., Westinghouse Electric Company, October 30, 2015. [http://www.iaea.org/inis/collection/NCLCollectionSto re/_Public/47/046/47046013.pdf?r=1]

2. S. Ray, E.L., F. Franceschini, Assessment of different materials for meeting the requirements of future fuel designs, in 2012 Reactor Fuel Performance Meeting. 2012: Manchester, UK.

3. Petrovic, B. Integral Inherently Safe Light Water Reactor (I2S-LWR) Concept: Extending SMR Safety Features to Large Power Output. in Proc. 2014 Intl. Congress on Advances in Nuclear Power Plants (ICAPP 2014). 2014. Charlotte, NC. become available to validate the model and to allow a proper estimation of the various parameters. Such experiments are currently under way at INL and expected to yield their first results in approximately three years.

\section{Acknowledgements}

The authors thank Dr. Hans Garkisch (consultant) and Dr. Paolo Ferroni (Westinghouse) for showing sustained interest in this work while it was being developed and for several useful discussions. The first author (MGM) thanks the Argentinean Chief of Cabinet of Ministers for their sustained financial support through the BEC.AR fellowship. This paper is derived wholly from a Georgia Tech thesis by M. G. Marquez developed entirely in residence at the Idaho National Laboratory under the direction of A. M. Ougouag. MGM thanks the INL for hosting him during two extended stays in which this research was performed. The second author (AMO) was supported in part by the $I^{2} S-L W R$ Nuclear Energy University Program Integrated Research Project (NEUP-IRP) from the U.S. DOE Office of Nuclear Energy. This source of funding is gratefully acknowledged. The research work and the bulk of the writing were performed at the Idaho National Laboratory: This manuscript has been co-authored by Battelle Energy Alliance, LLC under Contract No. DE-AC0705ID14517 with the U.S. Department of Energy. The United States Government retains and the publisher, by accepting the article for publication, acknowledges that the United States Government retains a nonexclusive, paid-up, irrevocable, world-wide license to publish or reproduce the published form of this manuscript, or allow others to do so, for United States Government purposes.

4. Was, G.S., Fundamentals of Radiation Materials Science. 2007: Springer.

5. Rest, J. and S.A. Zawadzki, FASTGRASS: A mechanistic model for the prediction of Xe, I, Cs, Te, $\mathrm{Ba}$, and $\mathrm{Sr}$ release from nuclear fuel under normal and severe-accident conditions. 1992: United States. p. 175.

6. Kinoshita, M., Towards the mathematical model of rim structure formation. Journal of Nuclear Materials, 1997. 248(0): p. 185-190.

7. Rest, J. and G.L. Hofman, Dynamics of irradiationinduced grain subdivision and swelling in $\mathrm{U}_{3} \mathrm{Si}_{2}$ and $\mathrm{UO}_{2}$ fuels. Journal of Nuclear Materials, 1994. 210(12): p. 187-202.

8. Rest, J. and G.L. Hofman, An alternative explanation for evidence that xenon depletion, pore formation, and grain subdivision begin at different local burnups. 
Journal of Nuclear Materials, 2000. 277(2-3): p. 231238.

9. Rest, J., A generalized model for radiation-induced amorphization and crystallization of $\mathrm{U}_{3} \mathrm{Si}$ and $\mathrm{U}_{3} \mathrm{Si}_{2}$ and recrystallization of $\mathrm{UO}_{2}$. Journal of Nuclear Materials, 1997. 240(3): p. 205-214.

10. Rest, J., Application of a mechanistic model for radiation-induced amorphization and crystallization of uranium silicide to recrystallization of $\mathrm{UO}_{2}$. Journal of Nuclear Materials, 1997. 248(0): p. 180-184.

11. Thomas, L.E., C.E. Beyer, and L.A. Chariot, Microstructural analysis of LWR spent fuels at high burnup. Journal of Nuclear Materials, 1992. 188(0): p. 80-89.

12. Spino, J., et al., Stereological evolution of the rim structure in PWR-fuels at prolonged irradiation: Dependencies with burn-up and temperature. Journal of Nuclear Materials, 2006. 354(1-3): p. 66-84.

13. Birtcher, R.C. and L.M. Wang, Stability of uranium silicides during high energy ion irradiation, in Materials Royal Society 1991 Fall Meeting. 1991: United States. p. 7.

14. Sandström, R., On recovery of dislocations in subgrains and subgrain coalescence. Acta Metallurgica, 1977. 25(8): p. 897-904.

15. Hansen, N. and D. Kuhlmann-Wilsdorf, Low energy dislocation structures due to unidirectional deformation at low temperatures. Materials Science and Engineering, 1986. 81(0): p. 141-161.

16. Nes, E., Recovery revisited. Acta Metallurgica et Materialia, 1995. 43(6): p. 2189-2207.

17. Rios, P.R., et al., Nucleation and growth during recrystallization. Materials Research, 2005. 8: p. 225238.

18. Sizmann, R., The effect of radiation upon diffusion in metals. Journal of Nuclear Materials, 1978. 69-70(0): p. 386-412.

19. Finlay, M.R., et al. Behaviour of irradiated uranium silicide fuel revisited. in 2002 International Meeting on Reduced Enrichment for Research and Test Reactors. 2002. San Carlos de Bariloche, Argentina.

20. Metzger, K.E., T.W. Knight, and R.L. Williamson, Model of U3Si2 Fuel System Using BISON Fuel Code, in Proceedings of ICAPP 2014. 2014: Charlotte, USA.

21. M.L. Bleiberg, R.M.B., B. Lustman. Proceedings on Radiation Damage in Reactor Materials. in Radiation Damage in Reactor Materials. 1963. Vienna.

22. Walker, C.T., et al., Concerning the microstructure changes that occur at the surface of $\mathrm{UO}_{2}$ pellets on irradiation to high burnup. Journal of Nuclear Materials, 1992. 188(0): p. 73-79.

23. Cunningham, M.E., M.D. Freshley, and D.D. Lanning, Development and characteristics of the rim region in high burnup UO2 fuel pellets. Journal of Nuclear Materials, 1992. 188(0): p. 19-27.

24. Une, K., et al., Microstructural change and its influence on fission gas release in high burnup UO2 fuel. Journal of Nuclear Materials, 1992. 188(0): p. 6572.

25. Nogita, K. and K. Une, Irradiation-induced recrystallization in high burnup UO2 fuel. Journal of Nuclear Materials, 1995. 226(3): p. 302-310.

26. Nogita, K. and K. Une, Radiation-induced microstructural change in high burnup UO2 fuel pellets. Nuclear Instruments and Methods in Physics Research Section B: Beam Interactions with Materials and Atoms, 1994. 91(1-4): p. 301-306.

27. Matzke, $\mathrm{H}$., On the rim effect in high burnup $U_{2} L W R$ fuels. Journal of Nuclear Materials, 1992. 189(1): p. 141-148.

28. Ray, I.L.F., H. Thiele, and H. Matzke, Transmission electron microscopy study of fission product behaviour in high burnup UO2, in Nuclear Materials for Fission Reactors, H.M. Schumacher, Editor. 1992, Elsevier: Oxford. p. 90-95.

29. Lassmann, K., et al., Modelling the high burnup $\mathrm{UO}_{2}$ structure in LWR fuel. Journal of Nuclear Materials, 1995. 226(1-2): p. 1-8.

30. Shimizu, H., The properties and irradiation behavior of $\mathrm{U}_{3} \mathrm{Si}_{2}$. 1965. p. Medium: ED; Size: Pages: 46.

31. Rest, J., Derivation of analytical expressions for the network dislocation density, change in lattice parameter, and for the recrystallized grain size in nuclear fuels. Journal of Nuclear Materials, 2006. 349(1-2): p. 150-159.

32. Woo, C.H. and B.N. Singh, Production bias due to clustering of point defects in irradiation-induced cascades. Philosophical Magazine A, Physics of Condensed Matter Defects and Mechanical Properties, 1992. 65(4): p. 889-912.

33. Dubinko, V.I., A.S. Abyzov, and A.A. Turkin, Numerical evaluation of the dislocation loop bias. Journal of Nuclear Materials, 2005. 336(1): p. 11-21.

34. Baranov, V.G., et al., Interaction of dislocations in $\mathrm{UO}_{2}$ during high burn-up structure formation. Journal of Nuclear Materials, 2014. 444(1-3): p. 129-137.

35. Baron, D., et al. Discussion about hbs transformation in high burn-up fuels. in Water Reactor Fuel Performance Meeting 2008. 2008. Korea, Republic of: KNS. see also Baron, D. et al, Nuclear Engineering and Technology, Vol.41 no.2 march 2009, pp.199-214 - special issue on the Water Reactor Fuel Performance Meeting 2008

36. Marlowe, M. O., High Temperature Isothermal Elastic Moduli of $\mathrm{UO}_{2}$, Journal of Nuclear Materials, 33 (1969) 242-244. 
37. Semenov, A.A. and C.H. Woo, Classical nucleation theory of microstructure development under cascadedamage irradiation. Journal of Nuclear Materials, 2003. 323(2-3): p. 192-204.

38. Woo, C.H., A.A. Semenov, and B.N. Singh, Analysis of microstructural evolution driven by production bias. Journal of Nuclear Materials, 1993. 206(2-3): p. 170199.

39. Rest, J., A model for the influence of microstructure, precipitate pinning and fission gas behavior on irradiation-induced recrystallization of nuclear fuels. Journal of Nuclear Materials, 2004. 326(2-3): p. 175184.

40. Johnson, R.A. and N.Q. Lam, Solute segregation in metals under irradiation. Physical Review B, 1976. 13(10): p. 4364-4375.

41. Ziegler, J. F., SRIM - The Stopping and Range of Ions in Matter, SRIM2013, http://www.srim.org/

42. Jonnet, J., et al., Growth mechanisms of interstitial loops in $\alpha$-doped UO2 samples. Nuclear Instruments and Methods in Physics Research Section B: Beam Interactions with Materials and Atoms, 2008. 266(1213): p. 3008-3012.

43. Bender, O. and P. Ehrhart, Self-interstitial atoms, vacancies and their agglomerates in electronirradiated nickel investigated by diffuse scattering of X-rays. Journal of Physics F: Metal Physics, 1983. 13(5): p. 911. 